\title{
Funnelling effect in networks
}

\author{
Parongama Sen \\ Department of Physics, University of Calcutta, \\ 92 Acharya Prafulla Chandra Road, \\ Calcutta 700009, India.
}

\begin{abstract}
Funnelling effect, in the context of searching on networks, precisely indicates that the search takes place through a few specific nodes. We define the funnelling capacity $f$ of a node as the fraction of successful dynamic paths through it with a fixed target. The distribution $D(f)$ of the fraction of nodes with funnelling capacity $f$ shows a power law behaviour in random networks (with power law or stretched exponential degree distribution) for a considerable range of values of the parameters defining the networks. Specifically we study in detail $D_{1}=D(f=1)$, which is the quantity signifying the presence of nodes through which all the dynamical paths pass through. In scale free networks with degree distribution $P(k) \propto k^{-\gamma}, D_{1}$ increases linearly with $\gamma$ initially and then attains a constant value. It shows a power law behaviour, $D_{1} \propto N^{-\rho}$, with the number of nodes $N$ where $\rho$ is weakly dependent on $\gamma$ for $\gamma>2.2$. The latter variation is also independent of the number of searches. On stretched exponential networks with $P(k) \propto \exp \left(-k^{\delta}\right), \rho$ is strongly dependent on $\delta$. The funnelling distribution for a model social network, where the question of funnelling is most relevant, is also investigated.
\end{abstract}

PACS numbers: $89.75 . \mathrm{Hc}, 89.75 . \mathrm{Fb}$

\section{INTRODUCTION}

Searching on networks has attracted a lot of attention recently. In general, the problem is to send a signal to a target node from a source node. It has been shown in some studies on real networks that it is possible to find short paths [1, 2, 3, 4, 5, 6, 7] during such a searching or navigation on small world and scale-free networks. This implies that even with only local knowledge of the network, there is a small world effect, i.e., the average number of steps to reach the target is $O(\log (N))$ where $N$ is the number of nodes in the network. In several theoretical studies the interest therefore has been to find out the scaling relation of the shortest searching path lengths with the number of nodes using different searching algorithms [8, 9, 10, 11, 12, 13, 14, 15, 16, 17, 18, 19, 20].

The notion of the small world effect emerged from the results of the original experiments made by Milgram [1] in which letters had to be hand delivered to a specific target. Apart from the observation of small world effect it was also claimed that the successful paths filtered through a few nodes [3] and this effect was termed funnelling. In these experiments however, very few chains were completed and the results could be less than conclusive. In a later study by Dodds et al [4], where search experiments on email networks were conducted, it was concluded that no such funnelling effect exists for social networks.

The question of funnelling has not been adequately addressed so far in any theoretical study to the best of our knowledge. In fact, no precise quantitative definition of funnelling has been proposed either.

We define the funnelling capacity of a node to be the fraction of dynamic paths through it when the target is fixed and the source is varied. In a realistic search, failure to reach the target has a considerable probability and searches with a possibility of termination [17, 18] have been studied earlier. Hence, with a fixed target, we define the funnelling capacity $f_{i}$ of the $i$ th node as

$$
f_{i}=\frac{\text { No of successful searches through the ith node }}{\text { Total no of successful paths }} .
$$

Defined in this way, it may seem that the funnelling capacity, averaged over all targets is the same as the betweenness centrality [21] of the node. The latter is defined as the fraction of shortest paths through a node and is a much studied quantity, but it must be remembered that it is obtained from the global knowledge of the network, and is thus a static property. Thus these two quantities are expected to behave differently in general. Funnelling capacity, which is a dynamic variable will obviously depend on the search algorithm.

It may also be mentioned that keeping the target fixed is an important criterion; a node in general is not expected to be part of the traffic for all choices of sourcetarget pairs (the target is selected randomly). On the other hand if one relaxes this criterion, only the hubs can show the funnelling effect. In the experiments of social searching also, funnelling has been considered by keeping the target fixed [1, 4].

In the present work, we have carried out simulations, in which, after generating the desired network, we fix a target and allow different nodes to be the source nodes. The dynamic path to the target (if it exists) is then found out to calculate the funnelling capacity. We then obtain the distribution $D(f)$, which is precisely the fraction of nodes with funnelling capacity $f$. To obtain $D(f)$, such searching processes are repeated on many networks.

Strictly speaking, $f_{i}$ is dependent on the target node as well, but here we have not studied that aspect directly. 
Rather, we expect that the dependence will be reflected in the distribution $D(f)$ itself.

The presence of funnelling effect would imply that $D_{1}=D(f=1)$ should be non-zero. We have therefore focussed our attention on this quantity and studied its behaviour as a function of the parameters of the network.

We have studied some random networks with given degree distribution as well as a correlated network which can serve as a toy model of a social network. The chosen degree distributions are either scale-free or stretched exponential type and are controlled by suitable parameters.

In addition, we consider as a parameter $\nu$, the ratio of the number of searches to be made (i.e., number of sources) to the total number of nodes $N$. Unless otherwise mentioned, the value of $\nu$ has been taken to be 0.1 .

Since the degree of a node is an important quantity of a network and it is customary to study the behaviour of quantities as a function of degree, we have also studied the average funnelling capacity $\langle f(k)\rangle$ of nodes with degree $k$ in case of scale-free networks.

We have used Monte Carlo simulations to study the funnelling effect by generating networks of size $\leq 5000$ and taking averages over typically 10000 to 20000 network configurations.

In sections II and III the results for scale-free and stretched exponential networks are presented respectively. In section IV, funnelling distribution in the toy model of social network has been discussed. In the last section we have summarised the results and drawn a few concluding remarks.

\section{FUNNELLING IN SCALE FREE NETWORKS}

In this section we discuss the results for networks which are constructed with a scale free degree distribution but are otherwise random. The search algorithm which has been used is degree based; such algorithms have been considered in networks (especially for scale free networks) quite commonly [9, 10].

We have generated random scale free networks with degree distribution $P(k) \propto k^{-\gamma}$ with the exponent $\gamma$ lying between 2 and 3 corresponding to realistic networks. The generation of the networks and the algorithm are described in detail in 17. We allow a minimum of two links (degree) for each node, while the maximum is $N^{1 / 2}$. All links are undirected and there are no multiple links between a given pair of nodes. We have used two degree based algorithms described in the following subsections. There is a general rule that a node can receive a message only once and searches terminate in case there is no neighbour left to whom the message can be passed.

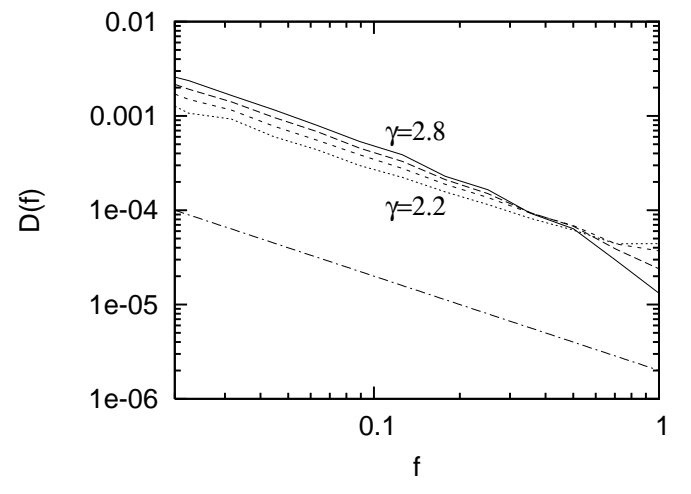

FIG. 1: The funnelling distribution $D(f)$ is shown for scalefree networks with $\gamma=2.8,2.6,2.4$ and 2.2 (from top to bottom) for $\nu=0.1$. The straight line has slope equal to -1 .

\section{A. Highest degree search (HDS)}

Here the message is passed to a neighbour with the highest degree $d_{h}$ (highest degree search or HDS). In case of multiple neighbouring nodes with degree $d_{h}$, one is selected randomly. However, if the target node happens to be a neighbour of a node, the message will be conveyed to it without considering the degree of other neighbours. When finding out the neighbour to whom the signal is to be passed, the neighbours which have already received it once are not considered.

With this algorithm, it is observed that $D(f)$ shows a power law decay against $f$ with exponent close to 1 but as $\gamma$ increases beyond $\gamma=\gamma^{*} \simeq 2.4$, the power law behaviour is observed only for a limited range of $f$ and shows a more rapid decay to zero as $f \rightarrow 1$. The results are shown in Fig. 1]

We find another intriguing behaviour of $D(f)$ for $\gamma$ values below $\gamma^{*}$. Here, $D(f)$ actually shows a tendency to increase for $f$ very close to unity. (By definition the maximum value of $f$ is one and therefore the increase in $D(f)$ cannot continue indefinitely. ) In fact, even for $\gamma>\gamma^{*}, f=1$ is a special point where $D(f)$ shows a significantly higher value than that at $f$ just below unity causing a discontinuity in $D(f)$. This is another reason to study the behaviour of $D_{1}$ more intricately. The reason for the discontinuity in $D(f)$ for $\gamma>\gamma^{*}$ is apparently due to the presence of a few nodes through which the searching path always passes (e.g., the nearest neighbours of the target).

In order to investigate the behaviour at $f=1$ more closely, we have plotted $D_{1}$ as a function of $\gamma$ (Fig. 2). We notice that $D_{1}$ first increases linearly with $\gamma$ and then tends to attain a constant value at higher $\gamma$.

The $\gamma=2.0$ is a special point where the average degree shows a logarithmic divergence. If the average degree is 


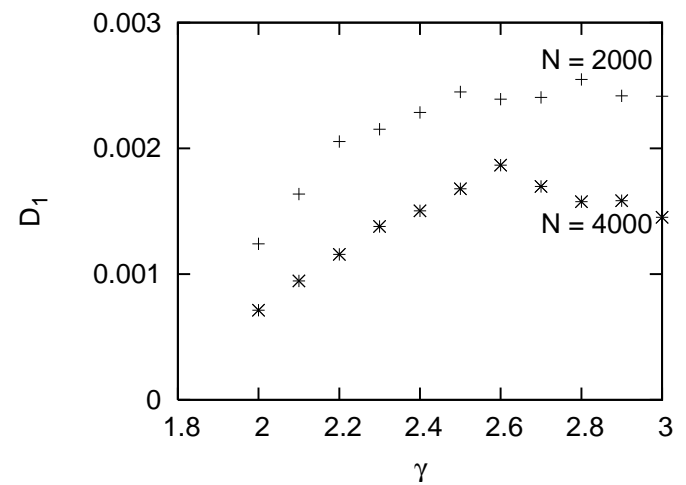

FIG. 2: The value of the distribution $D(f)$ at $f=1$ in scalefree networks is shown against $\gamma$ for two different network sizes.

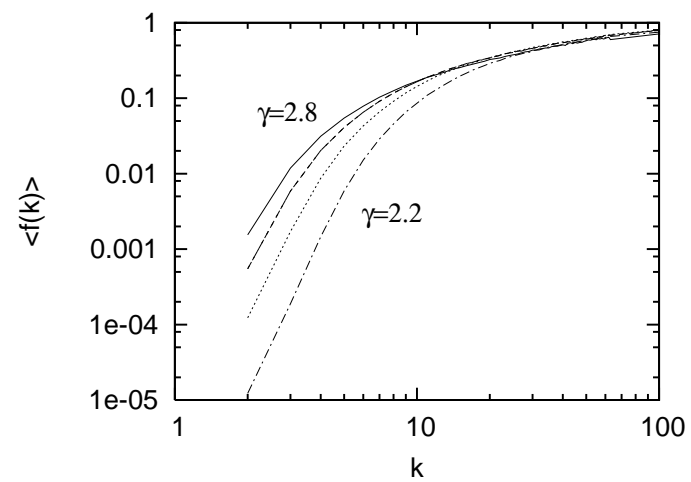

FIG. 3: The average funnelling capacity as a function of degree is shown for scale free networks with $\gamma=2.8,2.6,2.4$ and 2.2 .

large, $D_{1}$ will naturally be small as there are many available neighbouring nodes to pass on the signal. Hence the value of $D_{1}$ initially increases with $\gamma$. However, as $\gamma$ increases further the number of hubs decrease and consequently $D_{1}$ does not increase anymore. It can be expected that $D_{1}$ should decrease for very high values of $\gamma$, however, for $\gamma \leq 3.0$ this tendency is not strongly evident.

As $\gamma$ increases, the number of nodes with large degree becomes less and the above observation indicates that the funnelling capacity of nodes with less degree must increase with $\gamma$. Indeed, this is evident from a plot (Fig. 3) of the average funnellng capacity $\langle f(k)\rangle$ against degree $k$ for different $\gamma$. This data also show that there is no simple algebraic relation between $\langle f(k)\rangle$ and the degree as has been noted for the betweenness centrality showing clearly that betweenness centrality and funnelling are not trivially related.

Plotting $D_{1}$ against $N$, we show that funnelling disappears in the thermodynamic limit for all $\gamma$ (Fig. 4). $D_{1}$ in fact follows a power law decay with $N ; D_{1} \propto N^{-\rho}$. For $\gamma=2, \rho \simeq 0.75$ and decreases from this value as $\gamma$

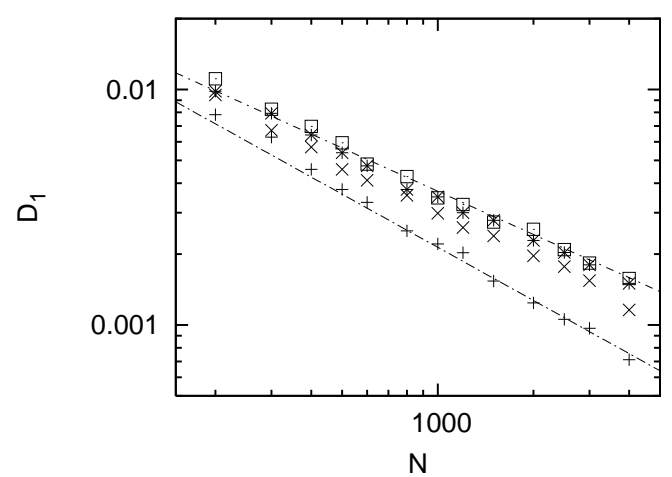

FIG. 4: The variation of $D_{1}$ against $N$ is shown for SFN with different $\gamma(\gamma=2.0,2.2,2.4$ and 2.8). At higher values of $\gamma$, the exponents are equal to $\sim 0.60$, while for $\gamma=2.0$ it is $\sim 0.75$.

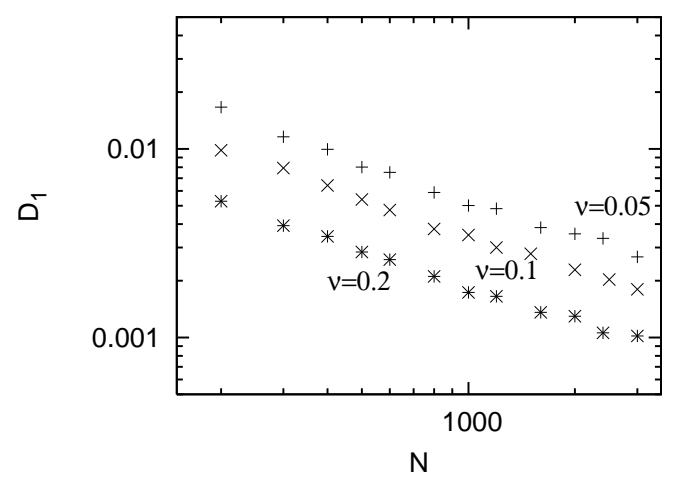

FIG. 5: The variation of $D_{1}$ against $N$ is shown for SFN with $\gamma=2.4$ and different values of $\nu$ showing that the exponents are not dependent on $\nu$.

is increased; beyond $\gamma \sim 2.2, \rho=0.60 \pm 0.01$ (weakly dependent on $\gamma$ ). This indicates that the funnelling capacity has universal behaviour for higher values of $\gamma$. Interestingly, the exponent is larger for smaller values of $\gamma$, i.e., when the number of hubs is large. This is consistent with the fact that in such a situation, there are multiple routes available for a message to reach the targeti, thereby making the funnelling capacity of nodes lesser.

We have also studied $D_{1}$ as a function of $N$ for different $\nu$ and find that power laws are obeyed for each $\nu$ (Fig. 5) with the exponent equal to $0.60 \pm 0.01$ in each case. The magnitude of $D_{1}$ decreases linearly with the number of search, which is also easy to understand (e.g., if there is only one searching process, all the nodes which take part in this search have funnelling capacity equal to 1 , the maximum possible value). 


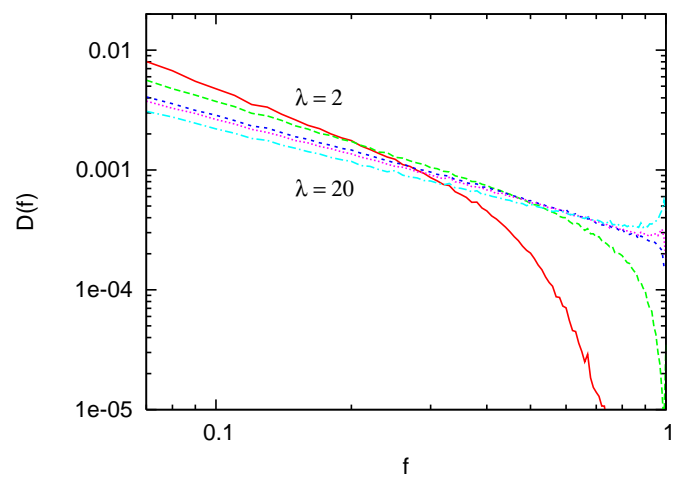

FIG. 6: The distribution $D(f)$ is shown for a scale free network with $\gamma=2.0$ with different algorithms corresponding to different values of $\lambda$ (eq (2) ).

\section{B. Tunable degree based algorithm}

Next we study the funnelling distributions on random scale-free networks with a tunable degree based algorithm. Precisely, here the search has a preferential algorithm. During the search, if one of the neighbours of the messenger node happens to be the target itself, the message will be sent to the target. If not, then the ith neighbour will receive the message with a probability $\Pi_{i}$, where

$$
\Pi_{i} \propto k_{i}^{\lambda} .
$$

Thus here the algorithm can be extended from a random search (RS) $(\lambda=0)$ to a highest degree search (HDS) $(\lambda \rightarrow \infty)$ scheme as described in 17]. The rule that a message cannot be passed on to the same node twice is still applied. The essential difference between the present algorithm and the HDS is while the tunable degree based algorithm is stochastic, the HDS is deterministic.

We have taken scale free networks with $\gamma<\gamma^{*}$ such that we know that in the limit $\lambda \rightarrow \infty$, it does show a funnelling effect (i.e., power law degree distribution up to $f=1$ ). We find that for small $\lambda$ values, $D(f)$ has a fast decay as $f$ approaches one, while above a certain value of $\lambda=\lambda^{*}$ it has a power law decay with the upward bend as noticed for the HDS. For $\lambda<\lambda_{c}$, there is a power law behaviour only over for a finite range of values of $f$. The value of $\lambda^{*}$ depends on $\gamma$, it being higher for higher values of $\gamma$. We find that $\lambda^{*}$ is in fact very high $(\sim 10$ for $\gamma=2.0$ ) (Fig. 6) and we have checked that at such large values of $\lambda$, essentially the signal is being passed to the neighbour having the highest degree. The power law decay of $D(f)$ for large $\lambda$ again occurs with an exponent close to unity, which is to be expected.

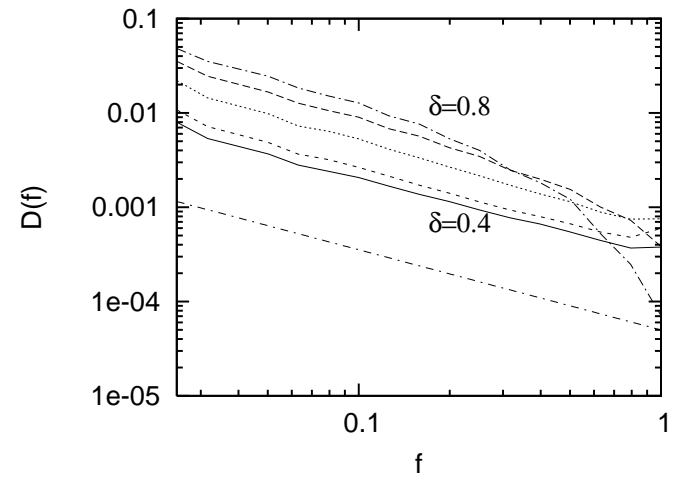

FIG. 7: The funnelling distribution $D(f)$ is shown for stretched exponential networks with $\delta=0.8,0.7,0.6,0.5$ and 0.4 (from top to bottom) with $\nu=0.1$. The straight line has slope equal to 0.85 .

\section{FUNNELLING IN STRETCHED EXPONENTIAL NETWORK}

There are many real world networks (e.g., social networks) which do not have a scale-free degree distribution. We have therefore considered networks in which the degree distribution has a stretched exponential distribution: $P(k) \sim \exp \left(-c k^{\delta}\right)$. The value of $c$ is unimportant and we set it equal to 1 . The maximum degree allowed here is $N^{1 / 2}$. Very small values of $\delta$ gives rise to a very highly connected network which is somewhat unphysical and therefore we have taken $\delta>0.2$. The funnelling distributions $D(f)$ again shows a power law variation with a change occurring at $\delta=\delta^{*}$ (lying between 0.6 and 0.7 ), above which the funnelling distribution falls rapidly with $f$. The power law exponent is, however, different from that observed in scale free networks; it has a value close to 0.85 . Once again we notice that below $\delta^{*}, D(f)$ shows a power law decay and a slightly upward bend as $f$ approaches one (Fig. 7).

Here too we study the variation of $D_{1}$ with network sizes and find that a power law variation exists, (Fig. 8) however, in this case the exponent is strongly dependent on $\delta$. The exponents decrease in magnitude as $\delta$ is increased, e.g., $\rho \simeq 1.0$ for $\delta=0.4$ and $\rho \simeq 0.5$ for $\delta=0.6$. Once again we note that as in the case of scale free networks, the exponent for the smaller value of $\delta$ is higher, when the number of highly connected nodes is larger.

\section{FUNNELLING IN A CORRELATED NETWORK}

Funnelling is an important issue in social searches and therefore we have considered in this section a network 


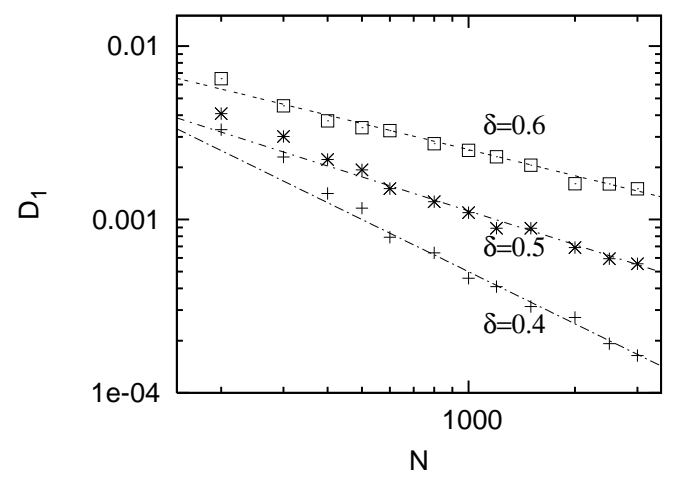

FIG. 8: $\quad D_{1}=D(f=1)$ against network size $N$ is shown for stretched exponential networks $\delta=0.6,0.5$ and 0.4 (from top to bottom) indicating that the exponents are different as the straight lines drawn to fit the curves have different slopes (see text).

which is not entirely random in the sense that there is a correlation between nodes. The nodes, in reality, have many characteristic features (other than the degree) which seriously affect the searching process [4, 13]. In a very simplified picture, we consider only one such characteristic which we call the similarity factor $\xi$ of the individuals, $\xi$ varying between 0 and 1 randomly. Since we have actually tried to simulate a social network, the degree distribution is taken to be $P(k) \propto \exp (-k)$. However, while constructing the network with such a distribution, the bonding between two nodes is now made according to the probability

$$
\mathcal{P}_{i, j} \propto\left|\xi_{i}-\xi_{j}\right|^{-\alpha}
$$

such that for positive values of $\alpha$, similar nodes will have more connection probability. As an example, $\xi$ may simply denote the geographical position of a node. The algorithm used is a greedy one: while searching, a node here sends the signal to a node with the similarity characteristic closest to that of the target node.

Searching on a generalised class of stretched exponential networks, (i.e., those with degree distribution $\left.\exp \left(-k^{\delta}\right)\right)$ with similarity dependent connections has been recently considered [19] and it has been observed that the success rate of searching is drastically reduced as $\delta$ is increased. Using a similarity based search algorithm and varying the parameter $\alpha$, it was observed that the best searchability occurs at values of $\alpha$ close to 1.5 for any $\delta$. For both higher and lower values of $\alpha$ the searchability deteriorates. At large $\alpha$, the network is highly clustered in the sense that nodes which have comparable values of $\xi$ happen to have a strong bonding and since the target node is randomly selected the success rate falls. On the other hand, at small $\alpha$, the nodes are highly uncorrelated which makes searching based on similarity ineffective.

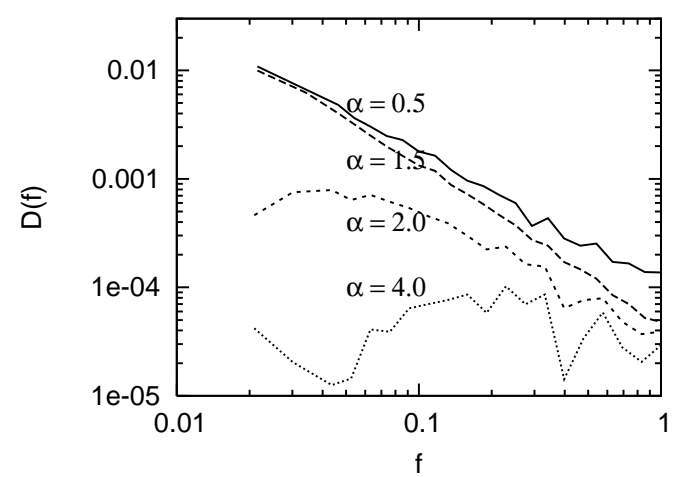

FIG. 9: The distribution $D(f)$ is shown for a network where the linkings depend on a similarity factor for different values of $\alpha$ (eq (3)).

As far as funnelling is concerned, we find some intriguing results which show that searchability and funnelling properties are not simply related. Here we have a considered a degree distribution $\propto \exp (-k)$ where the searchability is rather poor [19]. Still, for small values of $\alpha$, there is a power law variation of $D(f)$ with an exponent close to 1.5 (weakly dependent on $\alpha$ ). For higher values of $\alpha$, large fluctuations occur, the power law behaviour is lost and the distribution tends to become flat (see Fig. 9). The change in behaviour of the distribution might suggest that a phase transition is occurring here, but we would not like to conclude anything as the fluctuations are too large to comment.

In order to understand the above results, we first discuss the case $\alpha=0$ which corresponds to a network without any correlation. This is simply an exponential network where there is no funnelling effect with HDS as noted earlier. But with the present algorithm, we find that there is indeed a power law variation of $D(f)$. What could be the reason for this? It appears that since the nodes are uncorrelated, the target node is connected to nodes with arbitrary similarity factors. However not all of them will take part equally in the search process because of the algorithm and successful paths will be mostly through a few nodes making it possible the existence of a few nodes with large funnelling capacity.

Apparently, the funnelling effect diminishes with correlations as the present results suggest. In fact, with $\alpha \neq 0$, when we have a correlated network, there can be several nodes to which the signal can be passed which are equally 'distant' from the target. This effectively makes the funnelling capacity of individual nodes lesser which is reflected in the distribution. For very high $\alpha$, this effect is enhanced to a large extent making the distribution nearly flat. 


\section{DISCUSSIONS AND CONCLUDING REMARKS}

In this paper we have, for the first time to our knowledge, attempted a quantitative study of the phenomenon of funnelling relevant to search or navigation on a network. First we have proposed a definition of funnelling capacity $f$ of a node and thereafter estimated the funnelling distribution $D(f)$. The point $f=1$ has been treated specially as a non-zero value of $D_{1}=D(f=1)$ would indicate funnelling is indeed occurring. Our studies on scale-free and stretched exponential networks have shown that funnelling will not survive for infinite networks and also decrease if the number of searches is increased. However, we have obtained power law decay behaviour for both $D(f)$ versus $f$ and $D_{1}$ versus $N$ variations. The exponent for $D(f)$ is different for different networks. In case of the scale free network, we note that the exponent $\rho$ obtained from the $D_{1}$ versus $N$ plots is weakly dependent on $\gamma$ for $2.2<\gamma<3.0$ while in stretched exponential networks, it is non-universal. We have also used different algorithms in the different networks, e.g., degree based algorithms for networks which are uncorrelated and similarity based algorithm on correlated networks; the results show that the algorithm seriously affects the funnelling distribution.

To show how the algorithm can affect the funnelling capacity one can take the example of a simple hypothet- ical network. Suppose the network has uniform degree $k=l$ with a tree structure. Thus the successful paths to the target will flow with equal probability through its $l$ neighbouring nodes when the algorithm does not take into account any correlations (note that the HDS and the random search are identical in this case). Thus these $l$ nodes will take part in $1 / l$ fraction of searches, their $l^{2}$ neighbours also take part in $1 / l^{2}$ searches and so on making $D(f) \propto 1 / f$. However, if now an algorithm based on correlations is used, the message flow will no longer be uniform and the distribution $D(f)$ will be quite different. In fact we have obtained exponents for $D(f)$ which are different but of the order of unity in the different networks.

To explain the result obtained in [4] that there is no funnelling effect in a social search, one can argue on the basis of the present results that this is due to the fact that the human network is far from random and has quite strong correlations. On the other hand, in the earlier experiments [1], funnelling was observed since the number of searches conducted (compared to the network size) was very small.

Acknowledgment: Financial support from CSIR grant no. 3(1029)/05-EMR-II and UGC UPE (computational group) is acknowledged. Hospitality at Abdus Salam ICTP, Trieste, where part of the work was done, is also acknowledged.
[1] Milgram S.: The small world problem. Psychology Today 1, 60-67 (1967); Travers J.: and Milgram S., An Experimental Study of the Small World Problem. Sociometry 32, 425-443 (1969).

[2] Killworth P. D. and Bernard H. R.: The reversal smallworld experiment. Social Networks 1, 159-162 (1978).

[3] White H.C.: Search parameters for the small world problem. Social forces 49, 259-264 (1970).

[4] Dodds P. S., Muhamad R. and Watts D. J.: An experimental study of search in global social networks. Science 301, 827-829 (2003).

[5] Adamic L. A. and Adar E.: How to search a social network. Social Networks 27, 187-203 (2005).

[6] Clarke I., T. Hong, Miller S. G., Sandberg O. and Wiley B.: Protecting free expression online with Freenet. IEEE Internet Computing 6, 40-49 (2002).

[7] Liben_Nowell D., Novak J., Kumar R., Raghavan P. and Tomkins A.: Geographic routing in social networks. PNAS 102, 11623-11628 (2005).

[8] Kleinberg J.: Navigation in a Small World. Nature 406, 845 (2000).

[9] Adamic L. A., Lukose R. M., Puniyani A. R. and Huberman B. A.: Search in power-law networks. Phys. Rev. E 64, 046135 (2001).

[10] Kim B. J., Yoon C. N., Han S. K. and Jeong H.: Path finding strategies in scale-free networks. Phys. Rev. E 65, 027103 (2002).

[11] Zhu H. and Huang Z-X.: Navigation in a small world with local information. Phys. Rev. E 70, 036117 (2004).
[12] de Moura A. P. S., Motter A. E. and Grebogi C.: Searching in small-world networks. Phys. Rev. E 68, 036106 (2003).

[13] Watts D. J., Dodds P. S. and Newman M. E. J.: Identity and Search in Social Networks. Science 296, 1302-1305 (2002).

[14] Carmi S., Cohen R. and Dolev D.: Searching complex networks efficiently with minimal information. Europhys. Lett. 74, 1102-1108 (2006).

[15] Thadakamalla H. P., Albert R. and Kumara S. R. T.: Search in weighted complex networks. Phys. Rev. E 72, 066128 (2005).

[16] Clauset A. and Moore C.: How Do Networks Become Navigable. Preprint arxiv:cond-mat/0309415 (2003).

[17] Sen P.: A novel approach to study realistic navigations on networks. J. Stat. Mech. P04007 (2007).

[18] Basu Hajra K. and Sen P.: Effect of a static phase transition on searching dynamics. J. Stat. Mech. P06015 (2007).

[19] Sen P.: Realistic searches on stretched exponential networks. Pramana 71, 313-318 (2008).

[20] Serrano M. A., Krinkov D. and Boguna M.: SelfSimilarity of Complex Networks and Hidden Metric Spaces. Phys. Rev. Lett. 100, 078701 (2008).

[21] Freeman L. C.: A set of measures of centrality based on betweenness. Sociometry 40, 35-41 (1977); Goh K.I., Kahng B. and Kim D.: Universal Behavior of Load Distribution in Scale-Free Networks. Phys. Rev. Lett. 87, 278701 (2001). 